\title{
Thematic framework for exploring the use of a narrative CV, initial findings from secondary analysis of Royal Society data
}

\author{
$10^{\text {th }}$ January 2022
}

\author{
K Meadmore, A Recio-Saucedo, A Blatch-Jones, H Church, A Cross, K Fackrell, S Thomas, \\ L Tremain
}

National Institute for Health Research (NIHR) Evaluation, Trials and Studies Coordinating Centre, School of Healthcare Enterprise and Innovation, University of Southampton

Description: Here we present a thematic framework developed from secondary and document analysis of data collected by the Royal Society from their work on research culture and the subsequent development of the Résumé for Researchers. These data were shared with the NIHR to inform discussions about the potential for use of a narrative CV within the NIHR. A wider programme of work to explore the use of narrative CVs within the NIHR is currently underway.

Key words: narrative CV, research culture, secondary analysis, documentary analysis, NIHR, Royal Society, Résumé for Researchers

\section{Background}

In 2021, the NIHR joined other UK funders in a statement confirming their commitment to exploring a shared approach towards a narrative CV (see https://www.ukri.org/wpcontent/uploads/2021/07/UKRI-230721-4995CommsResumeJointFundersStatementFinal.pdf). The findings reported here are one element of a broader evaluation that is being undertaken by the NIHR to explore the use of narrative CVs within the NIHR.

\section{What we have done so far}

- We have undertaken secondary and document analysis of data shared with NIHR by the Royal Society from their work on research culture and the subsequent development of the Résumé for Researchers (https://royalsociety.org/topicspolicy/publications/2018/research-culture-embedding-inclusive-excellence/)

- Fifteen documents from workshops, feedback from consultations and reports were reviewed to explore whether information from the data gathered by the Royal Society could inform discussions about the potential use of a narrative CV within the NIHR

- Themes were extracted from the documents that were of interest from an NIHR perspective. Analysis focused on information that was most relevant to the NIHR context of using narrative CVs. It is anticipated that the concepts in the framework may also be of interest to other funders interested in exploring using narrative CVs. Here we present the thematic framework that resulted from this analysis.

\section{Thematic framework of key narrative CV areas of influence relevant to NIHR}

We developed a thematic framework that captures three broad areas relevant to NIHR (see Figure 1). The three areas were:

1. Challenges and impacts of implementing a narrative CV. This theme relates to practical concepts, benefits, and issues with using a narrative CV during which need to be considered if NIHR implemented a narrative CV approach. This theme includes guidelines for writing and assessing narrative CV content, how narrative CVs may affect equity and workload and, how it may work and align between and within funders.

2. Potential contributions of the narrative $\mathrm{CV}$ to wider research. This theme relates to considerations of wider research ecosystem challenges and impacts linked to 
actions which are specifically geared towards establishing a healthy (i.e., fair, transparent, efficient, inclusive, and diverse) research culture, and the role that narrative CVs may play if implemented by the NIHR.

3. Contexts and uses of the narrative CV. This theme relates to other potential uses of the narrative CV in the NIHR, such as promotion, hiring and appraisals.

Figure 1 shows the interconnections between the themes, and how the specific implementation issues sit within and interact with broader contextual issues regarding research culture.

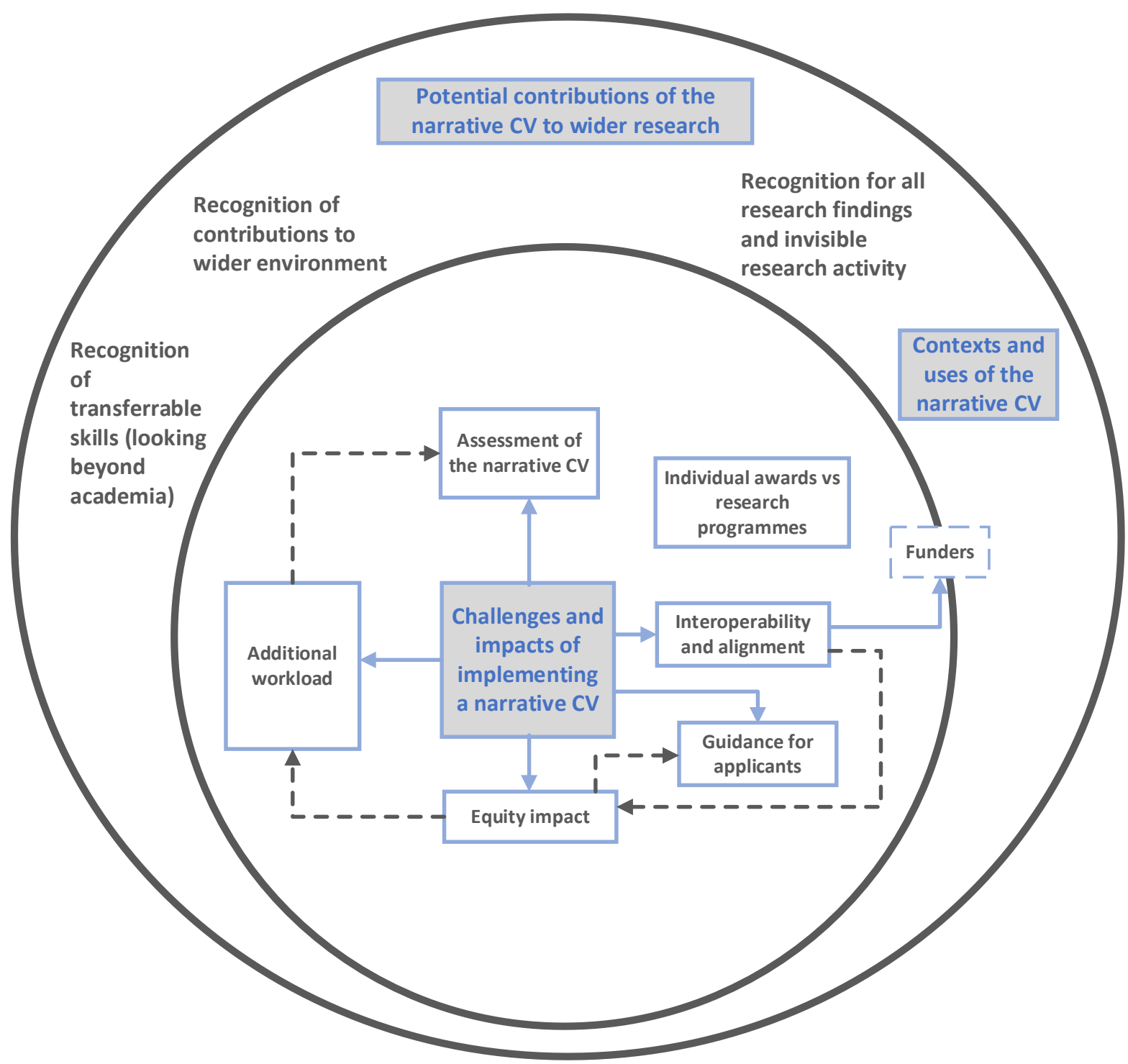

Figure 1. Thematic framework of considerations for funding organisations in the implementation of narrative CVs

Grey boxes represent three broad areas relevant to NIHR. Dark grey text represents the themes. The inner circle lists six themes linked to the challenges and impacts that the implementation of a narrative CV may have (shown by solid blue arrows). Dark grey, dashed arrows show links between themes. The outer circle includes three themes linked to research culture factors that influence the implementation or use of a narrative CV as well as other contexts and uses of the narrative CV. 


\section{What have we learned so far: Reflections for using a narrative CV}

Challenges and impacts for implementing a narrative CV. Considerations for implementation include:

- Provide clear and transparent guidance to reviewers on how to use and assess the information provided in a narrative CV to support and inform decision making appropriately, including how they should use any metrics provided within the narrative document

- Consider and evaluate the effect on workload both for applicants and reviewers, including assessing potential duplication in information

- Consider and evaluate the effects that the narrative CV may have on equity, in terms of career stage, diversity, gender, culture/ethnicity, and affiliation

- Provide clear guidance to applicants in terms of what narrative content is required and how it will be assessed, understanding of the individual versus team science tensions as well as guidance on the flexibility and weight of the different narrative modules. Examples of narrative CVs alongside guidelines should also be considered but caution is urged for gamification and so that these are not used as ideals

- Consider interoperability and alignment of the requirements for narrative CV with other funders and within NIHR programmes

- Analysis indicated that narrative CVs may lend themselves better to individual awards and assessment and consideration is needed for how the narrative CV would work for teams applying for research programmes.

Potential contributions of the narrative CV to wider research culture. The narrative CV may facilitate positive changes in the broader research ecosystem, including:

- Recognition of broad and valuable contributions to the wider research environment from applicants beyond traditional academic outputs

- Recognition of diverse career pathways into academia and associated transferable skills

- Recognition of 'invisible' research activity and wider acceptance of all research findings as valuable.

Contexts and uses of the narrative CV:

- The narrative CV could be considered in other contexts and for purposes different to research funding such as promotion, appraisals or hiring.

\section{What will happen next?}

Following development of the thematic framework, we conducted online focus groups and interviews to explore whether a narrative $\mathrm{CV}$ is an efficient and effective mechanism for the NIHR to collect and assess information (e.g., on track record, experience, and skills) on applicants and teams who apply for NIHR research programme and individual awards. Our work will inform strategic decision-making on assessment, review and funding decision processes. Findings from focus group and interviews, as well as secondary analysis will be brought together to provide an evidence-based understanding of perceptions about the use of a narrative CV in the NIHR. This analysis is part of a wider programme of work to explore the use of narrative CVs within the NIHR.

This work was conducted by the Research on Research team based at the NIHR Evaluation, Trials and Studies Coordinating Centre. No competing interests were disclosed.

Please contact ror@nihr.ac.uk for further information. 